\title{
Automated modification and fusion of voxel models to construct body phantoms with heterogeneous breast tissue: Application to MRI simulations
}

\author{
Joseph V. Rispoli*1,2,3, Steven M. Wright ${ }^{1,4}$, Craig R. Malloy ${ }^{5,6}$, Mary P. McDougall ${ }^{1,4}$ \\ ${ }^{1}$ Department of Biomedical Engineering, Texas A\&M University, College Station, Texas, United States of America \\ ${ }^{2}$ Weldon School of Biomedical Engineering, Purdue University, West Lafayette, Indiana, United States of America \\ ${ }^{3}$ School of Electrical and Computer Engineering, Purdue University, West Lafayette, Indiana, United States of America \\ ${ }^{4}$ Department of Electrical \& Computer Engineering, Texas A\&M University, College Station, Texas, United States of America \\ ${ }^{5}$ Advanced Imaging Research Center, University of Texas Southwestern Medical Center, Dallas, Texas, United States of America \\ ${ }^{6}$ Department of Radiology, University of Texas Southwestern Medical Center, Dallas, Texas, United States of America
}

Received: December 22, 2016

Accepted: February 15, 2017

Online Published: February 26, 2017

DOI: $10.5430 /$ jbgc.v7n1p1

URL: https://doi.org/10.5430/jbgc.v7n1p1

\begin{abstract}
Background: Human voxel models incorporating detailed anatomical features are vital tools for the computational evaluation of electromagnetic (EM) fields within the body. Besides whole-body human voxel models, phantoms representing smaller heterogeneous anatomical features are often employed; for example, localized breast voxel models incorporating fatty and fibroglandular tissues have been developed for a variety of EM applications including mammography simulation and dosimetry, magnetic resonance imaging (MRI), and ultra-wideband microwave imaging. However, considering wavelength effects, electromagnetic modeling of the breast at sub-microwave frequencies necessitates detailed breast phantoms in conjunction with whole-body voxel models.

Methods: Heterogeneous breast phantoms are sized to fit within radiofrequency coil hardware, modified by voxel-wise extrusion, and fused to whole-body models using voxel-wise, tissue-dependent logical operators. To illustrate the utility of this method, finite-difference time-domain simulations are performed using a whole-body model integrated with a variety of available breast phantoms spanning the standard four tissue density classifications representing the majority of the population.

Results: The software library uses a combination of voxel operations to seamlessly size, modify, and fuse eleven breast phantoms to whole-body voxel models. The software is publicly available on GitHub and is linked to the file exchange at MATLAB ${ }^{\circledR}$ Central. Simulations confirm the proportions of fatty and fibroglandular tissues in breast phantoms have significant yet predictable implications on projected power deposition in tissue.

Conclusions: Breast phantoms may be modified and fused to whole-body voxel models using the software presented in this work; user considerations for the open-source software and resultant phantoms are discussed. Furthermore, results indicate simulating breast models as predominantly fatty tissue can considerably underestimate the potential for tissue heating in women with substantial fibroglandular tissue.
\end{abstract}

Key Words: Electromagnetic fields, Anatomic models, Computer simulations, Magnetic resonance imaging, Breast

\footnotetext{
${ }^{*}$ Correspondence: Joseph V. Rispoli, Ph.D.; Email: jrispoli@purdue.edu; Address: Weldon School of Biomedical Engineering, Purdue University, West Lafayette, Indiana, 47907, United States of America. 


\section{INTRODUCTION}

Human voxel models incorporating detailed anatomical features are vital tools for the computational evaluation of electromagnetic (EM) fields within the body. ${ }^{[1]}$ One example research domain in which simulation of human voxel models has become a basis for ensuring patient safety is magnetic resonance imaging (MRI) studies. The IEC and FDA guidelines on maximum power deposition, quantified by specific absorption rate (SAR, with units $\mathrm{W} \mathrm{kg}^{-1}$ ), provide the basis for setting safe MRI scanner parameters controlling input power to the radiofrequency (RF) coil. ${ }^{[2,3]}$ To comply with these guidelines for human studies, it is common practice to characterize RF transmit coils and medical implants using full-wave electromagnetic modeling in order to establish the parameters that ensure safe power and SAR levels. ${ }^{[4]} \mathrm{Com}-$ monly, finite-difference time-domain (FDTD) based methods are utilized owing to the ease of incorporating SAR calculations with heterogeneous body models. ${ }^{[5-7]}$

In addition to whole-body human voxel models, phantoms representing regional anatomical features are often employed for EM simulations. While isolated regional phantoms suffice for certain applications, more thorough results may be obtained in some circumstances by fusing these smaller phantoms with a whole-body model. For example, localized breast voxel models have been developed for a variety of EM techniques utilizing short (relative to the body) wavelengths, including radiation dosimetry and ultra-wideband microwave imaging ${ }^{[8-11]}$ however, the frequencies currently associated with human MR scanners (i.e., $64-450 \mathrm{MHz}$ ) correspond to longer EM wavelengths with which the effects of the remainder of the body cannot be ignored. Thus, simulating RF field behavior during breast MRI scans would ideally incorporate a female whole-body voxel model into the FDTD mesh. Although several adult female body models are available to the research community, all are oriented in the standing or supine positions; ${ }^{[12-16]}$ unfortunately, these orientations limit the existing voxel models' applicability for simulating the filling factor of breast RF coils designed for women in the prone position, as is typically performed with MRI to avoid respiratory artifacts. ${ }^{[17,18]}$

Furthermore, the accuracy of breast phantoms is confounded by the anatomical variability of fatty (lipid) and fibroglandular tissues and their distinct material properties that affect electromagnetic exposure. ${ }^{[8]}$ Rudimentary efforts to model a prone breast either replaced or supplemented the body model with geometric volumes representing homogeneous breast tissues, ${ }^{[19,20]}$ while improved breast phantoms with heterogeneous fibroglandular and adipose tissue structures have been generated by using image data conversion $^{[11,21,22]}$ or software algorithms. ${ }^{[23-28]}$ Recognizing the need for anatomically-correct heterogeneous breast phantoms in conjunction with whole body voxel models, van der Velden et al. fused a body model with 3D image data acquired from five healthy volunteers and ultimately noted that the observed disparity of simulated SAR distributions among these models was due to indeterminate variations in size and tissue makeup. ${ }^{[29]}$ Breast size and tissue density have great variability among the patient population; to classify the extent of breast tissue density across women, radiologists largely have embraced the four tissue composition categories prescribed by the American College of Radiology (ACR) in the Breast Imaging Reporting and Data System (BI-RADS $\left.{ }^{\mathrm{R}}\right){ }^{\left[{ }^{30]}\right.}$ Using this method, breast density is defined as (a) almost entirely fat, (b) scattered fibroglandular tissue, (c) heterogeneous fibroglandular tissue, and (d) extreme fibroglandular tissue. An analysis of projected SAR encompassing these four classifications is used as a test bed in this study.

In order to address the need to augment existing whole-body voxel models to account for prone imaging and the necessity to use heterogeneous breast tissue models, this work details a software procedure to seamlessly modify and fuse heterogeneous breast phantoms to whole-body voxel models. First, the breast phantoms are resized and appropriately situated with respect to the whole-body voxel model, and subsequent voxel extrusion and subtraction operations effectively fuse the breast anterior to the pectoral muscles of the whole-body voxel models. To illustrate the utility of this method, we performed simulations of 7 tesla MRI radiofrequency excitation of a whole-body voxel model fused with nine high-resolution, anatomically-correct breast phantoms spanning the four BI-RADS tissue density classifications, thus demonstrating the importance of being able to modify the few existing whole body models for particular studies. Results confirm the proportions of lipid and fibroglandular tissues in breast phantoms have significant yet predictable implications on projected SAR. Finally, usage of the publiclyavailable software and resultant phantoms is discussed.

\section{METHODS}

\subsection{Voxel models and software}

Of the presently reported adult non-pregnant female wholebody models, "Ella" from the Virtual Population is the highest-resolution and most comprehensive tissue model of the adult female body. ${ }^{[12]}$ Disadvantages from the standpoint of breast modeling include the facts that Ella 1) represents a woman in the supine position and 2) does not include any fibroglandular tissue in the breast region. For this work, we adapted nine heterogeneous $0.5-\mathrm{mm}$ resolution pendant breast phantoms produced by the Hagness group at 
the University of Wisconsin-Madison (UW) for modeling microwave interactions between 0.5 and $20 \mathrm{GHz}{ }^{[11]}$ These phantoms were labelled according to the subject's radiologistassigned BI-RADS classification, denoted BI-RADS-a, -b, -c, and - d. $^{[30]}$ At least two phantoms were developed for each category, with the exception of a single phantom for BIRADS-d. All nine breast models included tissues defined as fatty, fibroglandular, transitional, and skin. Within the fatty and fibroglandular classes, the preceding Wisconsin-Calgary study of microwave dielectric properties identified increasing incongruence of conductivity and permittivity values above $500 \mathrm{MHz}$ and accordingly divided both tissue classes into three tiers, each defined by single-pole Cole-Cole and Debye relaxation models. ${ }^{[31]}$ As these discrepancies in dielectric properties have not been observed in the RF band, in this work the three tiers for each tissue type were merged into single definitions as fat or fibroglandular tissues. The fat fibroglandular, and skin tissues were assigned dielectric properties using the four-pole Cole-Cole equations stipulated by the IT'IS Foundation's tissue properties database. ${ }^{[32]}$ Voxels designated as transitional were assigned dielectric and physical properties averaging the values from breast fat and gland tissues. The resulting tissue dielectric properties at $7 \mathrm{~T}$ were $\sigma=0.033 \mathrm{~S} / \mathrm{m}$ and $\varepsilon_{r}=5.5$ for breast fat, $\sigma=$ $0.85 \mathrm{~S} / \mathrm{m}$ and $\varepsilon_{r}=62$ for breast gland, $\sigma=0.44 \mathrm{~S} / \mathrm{m}$ and $\varepsilon_{r}=34$ for transitional tissue, and $\sigma=0.64 \mathrm{~S} / \mathrm{m}$ and $\varepsilon_{r}=$ 50 for skin. For comparison to the approach of utilizing homogeneous phantoms, a hemispherical cylinder was also generated to represent the breast in the two extreme cases; that is, this geometry included a $1.5-\mathrm{mm}$ skin layer with the remainder uniformly assigned either fatty or fibroglandular tissue.

The volumetric tissue data from the nine image-derived phantoms ${ }^{[33]}$ were imported into MATLAB ${ }^{\circledR}$ (MathWorks, Natick, MA, USA). Since these voxel models only included heterogeneous tissue from a single breast, with the thorax modeled as uniform two-dimensional slabs of skin, fat, and muscle, modifications were necessary to enable seamless integration with the Ella body model. The voxels from the thorax slabs were removed and each phantom was duplicated for bilateral breast simulation. The breast phantoms were then enlarged using a nearest-neighbor interpolation routine to achieve a maximum $13.3-\mathrm{cm}$ diameter in order to equally fill the RF coil. These dimensions were selected as a worst case to position the breast tissue $10 \mathrm{~mm}$ from the nearest coil conductor, as is the case with the actual RF coil. The resulting size of each breast phantom may be adjusted by providing the software routine with input parameters defining the desired dimensions. The finest-resolution Ella v1.3 voxel model, with $0.5-\mathrm{mm}$ isotropic resolution, was imported into MATLAB for joining to the $0.5-\mathrm{mm}$ breast phantoms. In accord with recent studies of MRI data segmentation between the breast and the pectoral muscle, the anterior extent of Ella's chest wall remained flat and aligned on the coronal plane. ${ }^{[34,35]}$ To position the breast phantoms on the Ella model before the fusing operation detailed below, two voxels were manually selected for centering the posterior coronal layer of all left and right breast phantoms. These locations were determined by orienting the base of each breast phantom on the inmost posterior coronal plane in Ella's anterior thorax that solely overlapped breast tissue, fat, and skin, i.e., without encroaching on the pectoral fascia or adjacent muscle tissue. As a result, the medial extent of each breast phantom's base was situated underneath Ella's skin layer, whereas the lateral extent of the base extended in free space above the anterior skin layer, as shown in Figure 1a. The locations for positioning the breast phantoms may be revised by providing the software function alternate coordinates with respect to the body phantom.
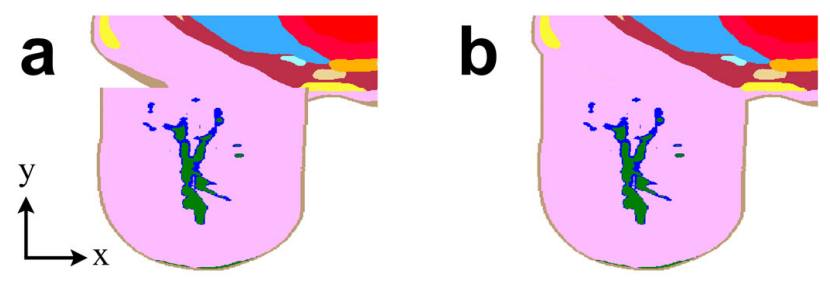

Figure 1. Renderings of breast phantom modification and fusion with whole-body voxel model

Two-dimensional axial mesh slices illustrate the (a) initial discontinuity and (b) subsequent joining of BI-RADS-a UW Phantom 1 and Ella, with skin tissue labelled brown, glandular tissue green, transitional tissue blue, and fatty tissues pink and yellow.

To automate integration of breast phantoms with a body model, we developed a software function to extrude and trim voxels from each breast phantom. First, voxels on the posterior coronal layer separated from Ella by free space were individually extruded toward the body model, penetrating the skin layer until encountering a voxel of adjacent tissue. By the same token, breast phantom voxels defined as skin that were situated inside the Ella phantom were removed from the phantom. Any remaining voxels from the heterogeneous breast phantoms that were co-located with the body model took meshing precedence in simulations, overwriting Ella voxels assigned skin, breast (fat), subcutaneous adipose tissue, and average infiltrated fat. These extrusion and trimming functions were applied independently for both the left and right breast positions. To illustrate this integration, the Ella voxel model fused with BI-RADS-b UW Phantom 2 is illustrated in Figure 1b. 


\subsection{Simulations}

\subsubsection{Mesh setup}

All breast phantoms were exported in a file format to facilitate import into commercial FDTD software (XFdtd ${ }^{\circledR}$ 7.4, Remcom, State College, PA, USA). A previously-described quadrature ${ }^{1} \mathrm{H}$ breast volume coil (as reported in Ref. ${ }^{[19]}$ ) was positioned over each of the 11 right breast phantoms. As illustrated in Figure 2, the coil conductors were centered on the breast phantom and separated from the nearest skin voxel by $10 \mathrm{~mm}$, as is the case in the actual coil former. The coil was driven by two sinusoidal feeds with appropriate $90^{\circ}$ phase shifts to simulate quadrature operation. The frequency was set to $298 \mathrm{MHz}$ to model operation at $7 \mathrm{~T}$; the simulation frequency may be adjusted to model other field strengths, e.g., $128 \mathrm{MHz}$ for $3 \mathrm{~T}$. To account for the different impedances of the quadrature pair, current source feed amplitudes were adjusted to deliver equal input power to the two quadrature channels with less than $0.5 \%(-23 \mathrm{~dB})$ variation. Cell gridding was adaptively set between 0.5 and $1.0 \mathrm{~mm}$ for the coil and breast phantom, and all curved conductor geometries utilized the software's conformal meshing capabilities. The entire mesh was surrounded by a quarter-wavelength of free space padding cells and the boundary comprised seven perfectly matched layers. Simulations were run on a workstation with two linked NVIDIA GeForce GTX 780 GPU cards, each with 3 GB RAM and 2,304 cores, resulting in an average computational time of 73 minutes. Convergence was determined by transients dissipating to $-50 \mathrm{~dB}$ deviation from the pure sinusoidal wave. Steady-state field data were calculated for all tissue voxels interior to the RF coil and globally on a central sagittal plane, and SAR data were calculated throughout the phantoms.

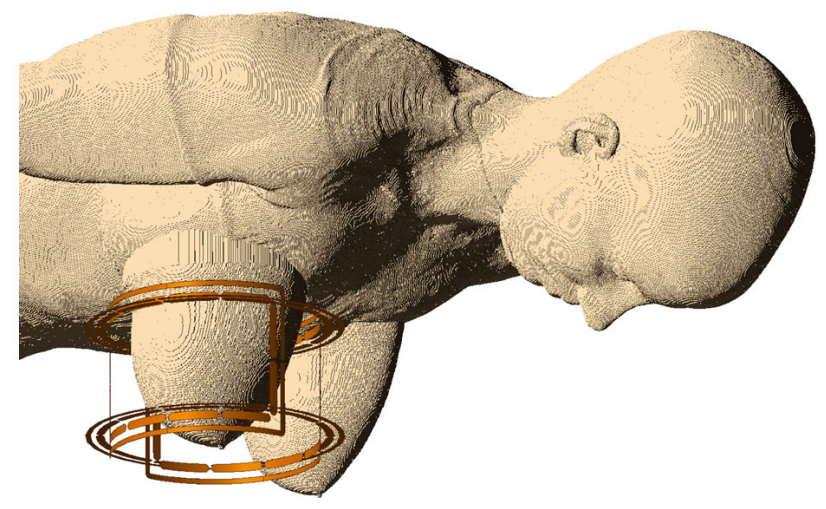

Figure 2. The Ella voxel model fused with BI-RADS-c UW Phantom 2, together surrounded by the quadrature volume breast coil

\subsubsection{Analysis}

Steady-state data output from FDTD simulations were imported into MATLAB for analysis. Mean transmit-field mag- netic flux density $\left(\mathbf{B}_{1}{ }^{+} \mid\right.$, with units $\left.\mathrm{T}\right)$ was computed inside the volume RF coil; only tissue voxels were included and voxel size was taken into account during averaging. As MR scanners typically monitor SAR with respect to the transmit magnitude, all SAR results in this study were scaled to achieve an average $\left|\mathbf{B}_{1}{ }^{+}\right|$of $1 \mu \mathrm{T}$ at $100 \%$ duty cycle. Accordingly, the raw and 10-g spatial-average SAR for each case were gauged in watts per kilogram per squared microtesla $\left(\mathrm{W} / \mathrm{kg} / \mu \mathrm{T}^{2}\right)$. To assess relative transmit coil power requirements as it relates to breast density, the net input (i.e., forward minus reflected) power required to achieve average $\left|\mathbf{B}_{1}{ }^{+}\right|$of $1 \mu \mathrm{T}$ was also noted.

\section{RESULTS}

Figure 3 displays raw and 10-g average SAR plots on the central sagittal slice. As anticipated, the raw SAR plots reveal localized maxima in regions with higher conductivity and permittivity as compared to adipose tissue. Notably, all BI-RADS-c and -d phantoms resulted in maximum 10$g$ average SAR incorporating gland regions interior to the breast, whereas the maxima in all BI-RADS-a, -b, and uniform phantoms were in the skin layer on the breast surface. Furthermore, note the higher-density phantoms are more inclined to have internal SAR maxima as opposed to surface localization in the mostly-fatty cases.

SAR data and coil efficiency for all cases are shown in Table 1. Compared to the mostly-fatty BI-RADS-a UW phantoms, the maximum local 10-g average SAR was threefold higher in the denser BI-RADS-d UW Phantom 1. Note the extreme case of a uniform cylinder of dense gland tissue resulted in almost fourfold the SAR of the otherwise worst-case BIRADS-d UW Phantom 1. As expected with more substantial dielectric loads, coil transmit efficiency decreased with denser breast phantoms, as indicated by the increased power requirements to achieve an average $\mid \mathbf{B}_{1}{ }^{+}$I of $1 \mu \mathrm{T}$ throughout the breast.

\section{Discussion}

The MATLAB functions developed for this study collectively facilitated 1) three-dimensional resizing, 2) placement with respect to the whole-body voxel model, and 3) voxel extrusion and subtraction to fuse all peripheral breast phantoms with the whole-body voxel model. In this study, the functions were performed on breast phantoms to illustrate the utility of fusing anatomical voxel models. The software may be applied to other regions, e.g., tumors, patient-specific features, or richly-detailed extremity phantoms. As an added benefit of this software method, the fusing operation does not modify the body model itself, nor require distribution with the body model. Therefore, any resulting secondary 
phantoms may be independently distributed under separate licensing terms. The software developed for this work is open-source, publicly-available in the GitHub repository, and linked to the file exchange at MATLAB Central. ${ }^{[36]}$ The bundled readme documentation details the function names and descriptions, input/output variables, and recommended execution sequence.

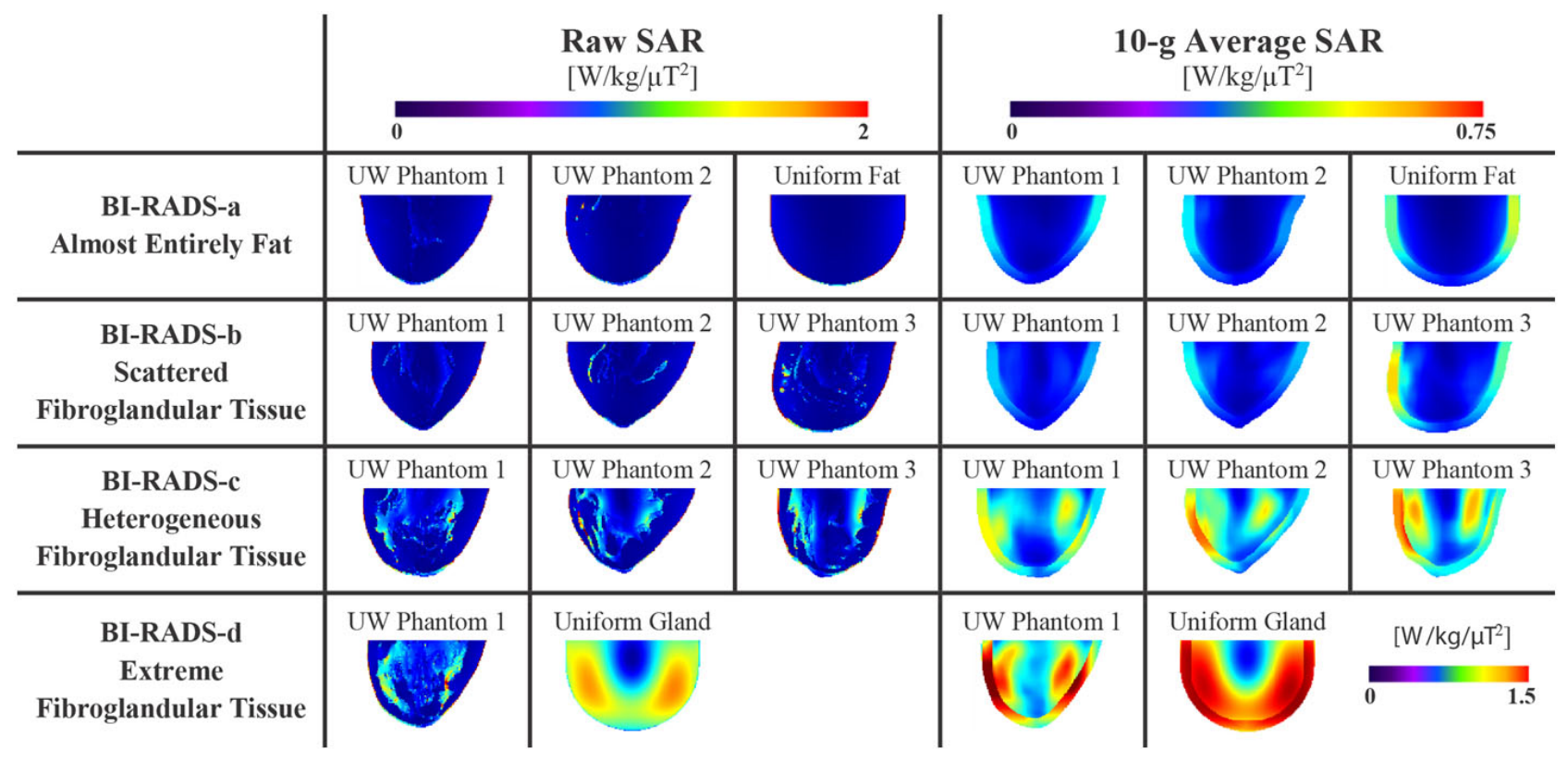

Figure 3. Raw and 10-g average SAR plots through the right breast's central sagittal slice for the 11 phantom cases; extreme average SAR in the uniform glandular phantom required a separate scale bar for the single case

Table 1. Coil maximum 10-g average SAR and power efficiency data

\begin{tabular}{llll}
\hline Breast Phantom & Tissue Classification & $\begin{array}{l}\text { Maximum 10-g Average SAR } \\
{\left[\mathbf{W} / \mathbf{k g} / \boldsymbol{\mu} \mathbf{T}^{2}\right]}\end{array}$ & $\begin{array}{l}\text { Input Power } \\
{[\mathbf{W}]+}\end{array}$ \\
\hline BI-RADS-a UW Phantom 1 & almost entirely fat & 0.30 & 0.63 \\
BI-RADS-a UW Phantom 2 & almost entirely fat & 0.30 & 0.64 \\
Uniform fat with skin layer & almost entirely fat & 0.45 & 0.67 \\
BI-RADS-b UW Phantom 1 & scattered fibroglandular tissue & 0.25 & 0.62 \\
BI-RADS-b UW Phantom 2 & scattered fibroglandular tissue & 0.30 & 0.65 \\
BI-RADS-b UW Phantom 3 & scattered fibroglandular tissue & 0.50 & 0.69 \\
BI-RADS-c UW Phantom 1 & heterogeneous fibroglandular tissue & 0.61 & 0.72 \\
BI-RADS-c UW Phantom 2 & heterogeneous fibroglandular tissue & 0.58 & 0.68 \\
BI-RADS-c UW Phantom 3 & heterogeneous fibroglandular tissue & 0.69 & 0.72 \\
BI-RADS-d UW Phantom 1 & extreme fibroglandular tissue & 0.89 & 0.75 \\
Uniform gland with skin layer & extreme fibroglandular tissue & 3.4 & 1.8 \\
\hline
\end{tabular}

The simulation study findings demonstrated significant variation among breast phantoms with varying levels of fat and fibroglandular breast tissues. During RF transmission, breast tissues with higher electrical conductivity and permittivity values, i.e., skin and glands, intensify the electric field and consequently are subject to greater localized SAR. For instance, a dense heterogeneous (BI-RADS-d) breast model produced threefold higher maximum 10-g average SAR as compared to mostly fatty breasts (BI-RADS-a). The geometric phantoms representing homogeneous tissue illustrated the expected RF field interference patterns in a uniform, electrically large dielectric. ${ }^{[37]}$ The intensity of this effect was especially apparent with the homogeneous gland tissue phantom and its symmetric SAR distributions as shown in Figure 
3 ; thus, the suitability of uniform phantoms for modeling in vivo breast scans is problematic. This study's findings indicate simulating the breast as predominantly-fatty tissue can considerably underestimate the potential for tissue heating in women with extensive fibroglandular breast tissue. This line of reasoning suggests to conservatively characterize SAR for regulatory compliance, simulation analyses of breast coils should incorporate a heterogeneous breast model largely consisting of fibroglandular tissue.

To facilitate a straightforward comparison, this study assumed equivalent breast sizes and positioning within the RF coil structure. Future research may provide further insight into the effects on SAR with varying breast size and positioning. This study was also limited to a single validated coil design; simulations featuring other RF coils may be informative. Finally, this simulation study quantified SAR, which is currently the standard metric for safety regulatory compliance. Thermometry studies may further complement the simulated SAR data for predicting tissue temperature rise.

This work addressed two problems relevant to modeling the breast accurately - the need to augment existing body voxel models to account for prone imaging and to use heterogeneous breast tissue models. The software developed to fuse breast and body phantoms is publicly available and may be employed to join other anatomical features. The ensuing breast MRI simulation study presented herein incorporated several high-resolution, anatomically-correct breast phantoms. Simulation results indicate breasts with predominantly fibroglandular tissue are susceptible to significantly increased SAR as compared to breasts mostly composed of fat.

\section{ACKNOWLEDGEMENTS}

This work was supported in part by CPRIT contract RP100625 and NIH awards R21EB016394 and P41EB015908.

\section{REFERENCES}

[1] Hand JW. Modelling the interaction of electromagnetic fields (10 $\mathrm{MHz}-10 \mathrm{GHz}$ ) with the human body: methods and applications. Phys Med Biol. 2008; 53: R243-R286. https ://doi .org/10.1088/00 31-9155/53/16/R01

[2] Technical committee 62 (Electrical equipment in medical practice), subcommittee 62B (Diagnostic imaging equipment). Medical electrical equipment-Part 2-33: Particular requirements for the safety of magnetic resonance equipment for medical diagnosis. $3.1 \mathrm{ed}$. Geneva (Switzerland): International Electrotechnical Commission; 2013. Report No. 60601-2-33.

[3] Center for Devices and Radiological Health. Guidance for the submission of premarket notifications for magnetic resonance diagnostic devices. Rockville (MD): Food and Drug Administration; 2016. Report No. 340 .

[4] Nyenhuis J. RF device safety and compatibility. eMagRes. 2010 https://doi.org/10.1002/9780470034590.emrstm1160

[5] Collins CM, Li S, Smith MB. SAR and B1 field distributions in a heterogeneous human head model within a birdcage coil. Magn Reson Med. 1998; 40: 847-856. https ://doi.org/10.1002/mr m. 1910400610

[6] Dimbylow P. FDTD calculations of the whole-body averaged SAR in an anatomically realistic voxel model of the human body from $1 \mathrm{MHz}$ to $1 \mathrm{GHz}$. Phys Med Biol. 1997; 42: 479-490. https: //doi.org/10.1088/0031-9155/42/3/003

[7] Jin J, Chen J. On the SAR and field inhomogeneity of birdcage coils loaded with the human head. Magn Reson Med. 1997; 38: 953-963. https://doi.org/10.1002/mrm. 1910380615

[8] Dance DR, Skinner CL, Alm Carlsson G. Breast dosimetry. Appl Radiat Isot. 1999; 50: 185-203. https://doi.org/10.1016/S0 969-8043 (98) 00047-5

[9] Hassan AM, El-Shenawee M. Review of electromagnetic techniques for breast cancer detection. IEEE Reviews in Biomedical Engineering. 2011; 4: 103-118. https : //doi .org/10.1109/RBME. 2011. 2169780
[10] Parker MS, Hui FK, Camacho MA, et al. Female breast radiation exposure during CT pulmonary angiography. Am J Roentgenol. 2005; 185: 1228-1233. https://doi.org/10.2214/AJR.04.0770

[11] Zastrow E, Davis SK, Lazebnik M, et al. Development of anatomically realistic numerical breast phantoms with accurate dielectric properties for modeling microwave interactions with the human breast. IEEE T Bio-Med Eng. 2008; 55: 2792-2800. https : //doi.org/10.1109/TBME. 2008.2002130

[12] Christ A, Kainz W, Hahn EG, et al. The Virtual Family-development of surface-based anatomical models of two adults and two children for dosimetric simulations. Phys Med Biol. 2010; 55: N23-N38. https : //doi .org/10.1088/0031-9155/55/2/N01

[13] Dimbylow P. Development of the female voxel phantom, NAOMI, and its application to calculations of induced current densities and electric fields from applied low frequency magnetic and electric fields. Phys Med Biol. 2005; 50: 1047-1070. https ://doi.org/10.108 8/0031-9155/50/6/002

[14] Liu W, Collins CM, Smith MB. Calculations of B1 distribution, specific energy absorption rate, and intrinsic signal-to-noise ratio for a body-size birdcage coil loaded with different human subjects at 64 and $128 \mathrm{MHz}$. Appl Magn Reson. 2005; 29: 5-18. https : //doi .org/10.1007/BF03166953

[15] Nagaoka T, Watanabe S, Sakurai K, et al. Development of realistic high-resolution whole-body voxel models of Japanese adult males and females of average height and weight, and application of models to radio-frequency electromagnetic-field dosimetry. Phys Med Biol. 2004; 49: 1-15. https://doi.org/10.1088/0031-9155/49/1 1001

[16] Stabin MG, Emmons MA, Segars WP, et al. Realistic reference adult and paediatric phantom series for internal and external dosimetry. Radiat Prot Dosim. 2012; 149: 56-59. https://doi.org/10.109 $3 /$ pd/ncr383

[17] Orel SG, Schnall MD. MR imaging of the breast for the detection, diagnosis, and staging of breast cancer. Radiology. 2001; 220: 13-30. https://doi.org/10.1148/radiology.220.1.r01j13113 
[18] Hylton NM, Frankel SD. Imaging techniques for breast MR imaging. Magn Reson Imaging Clin N Am. 1994; 2: 511-525. PMid: 7489305.

[19] McDougall MP, Cheshkov S, Rispoli J, et al. Quadrature transmit coil for breast imaging at 7 tesla using forced current excitation for improved homogeneity. J Magn Reson Imaging. 2014; 40: 1165-1173. https://doi.org/10.1002/jmri.24473

[20] Winkler SA, Rutt BK. Practical methods for improving B1+ homogeneity in 3 tesla breast imaging. J Magn Reson Imaging. 2015; 41: 992-999. https://doi.org/10.1002/jmri. 24635

[21] Klifa C, Carballido-Gamio J, Wilmes L, et al. Quantification of breast tissue index from MR data using fuzzy clustering. Proceedings of the 26th Annual International Conference of the IEEE Engineering in Medicine and Biology Society; 2004 Sep 1-5, San Francisco, CA. Piscataway (NJ): IEEE; c2004. p. 1667-1670. https : //doi.org/10.1109/IEMBS. 2004.1403503

[22] Samani A, Bishop J, Yaffe MJ, et al. Biomechanical 3-D finite element modeling of the human breast using MRI data. IEEE T Bio-Med Eng. 2001; 20: 271-279. https://doi.org/10.1109/42.92147 6

[23] Bliznakova K, Bliznakov Z, Bravou V, et al. A three-dimensional breast software phantom for mammography simulation. Phys Med Biol. 2003; 48(22): 3699. https://doi.org/10.1088/0031-9 $155 / 48 / 22 / 006$

[24] Bliznakova K, Sechopoulos I, Buliev I, et al. BreastSimulator: A software platform for breast x-ray imaging research. J Biomed Graph Comput. 2012; 2(1): 1. https://doi.org/10.5430/jbgc.v2n $1 \mathrm{p} 1$

[25] Bakic PR, Zhang C, Maidment ADA. Development and characterization of an anthropomorphic breast software phantom based upon region-growing algorithm. Med Phys. 2011; 38: 3165-3176. https://doi.org/10.1118/1.3590357

[26] Chen B, Shorey J, Saunders Jr RS, et al. An Anthropomorphic Breast Model for Breast Imaging Simulation and Optimization. Acad Radiol. 2011; 18(5): 536-546. https://doi.org/10.1016/j.acra. 201 0.11 .009

[27] Li CM, Segars WP, Tourassi GD, et al. Methodology for generating a 3D computerized breast phantom from empirical data. Med Phys. 2009; 36: 3122-3131. https://doi .org/10.1118/1.3140588
[28] Ma AKW, Gunn S, Darambara DG. Introducing DeBRa: a detailed breast model for radiological studies. Phys Med Biol. 2009; 54(14): 4533. https://doi .org/10.1088/0031-9155/54/14/010

[29] van der Velden TA, Italiaander M, van der Kemp WJM, et al. Radiofrequency configuration to facilitate bilateral breast 31P MR spectroscopic imaging and high-resolution MRI at 7 tesla. Magn Reson Med. 2015; 74: 1803-1810. https://doi.org/10.1002/mrm.25 573

[30] Morris E, Comstock C, Lee C. ACR BI-RADS $®$ Atlas, Breast Imaging Reporting and Data System. Reston (VA): American College of Radiology; c2013. ACR BI-RADS $®$ magnetic resonance imaging; p. 1-165.

[31] Lazebnik M, Popovic D, McCartney L, et al. A large-scale study of the ultrawideband microwave dielectric properties of normal, benign and malignant breast tissues obtained from cancer surgeries. Phys Med Biol. 2007; 52: 6093-6115. https : //doi .org/10.1088/00 31-9155/52/20/002

[32] Hasgall P, Neufeld E, Gosselin M, et al. IT'IS database for thermal and electromagnetic parameters of biological tissues. Zurich (Switzerland): IT'IS Foundation, c2013. http://dx.doi.org/10.13099/ ViP-Database-V2.4

[33] Zastrow E, Davis SK, Lazebnik M, et al. Database of 3D gridbased numerical breast phantoms for use in computational electromagnetics simulations. Madison (WI): University of WisconsinMadison; c2014 [cited 2014 Sep 21]. Available from: https: //uwcem.ece.wisc.edu/phantomRepository.html

[34] Ertas G, Doran SJ, Leach MO. A computerized volumetric segmentation method applicable to multi-centre MRI data to support computeraided breast tissue analysis, density assessment and lesion localization. Med Biol Eng Comput. 2016. https://doi.org/10.1007/ s11517-016-1484-y

[35] Mustra M, Grgic M, Rangayyan RM. Review of recent advances in segmentation of the breast boundary and the pectoral muscle in mammograms. Med Biol Eng Comput. 2016; 54: 1003-1024. https://doi.org/10.1007/s11517-015-1411-7

[36] Rispoli J. Breast-body-fusion [software]. San Francisco (CA): GitHub; c2015 [cited 2015 Apr 14]. Available from: https:// github.com/jrispoli/breast-body-fusion

[37] Yang QX, Wang J, Zhang X, et al. Analysis of wave behavior in lossy dielectric samples at high field. Magn Reson Med. 2002; 47: 982-989. https://doi.org/10.1002/mrm. 10137 